\title{
HYDROPHOBICITY OF WATER AT THE SURFACE AS STUDIED BY LASER-INDUCED FLUORESCENCE MICROSCOPY
}

\author{
YAO-QUN LI ${ }^{\mathrm{a}}{ }^{*}$, SHINYA SASAKI $^{\mathrm{b}}$, \\ TAKANORI INOUE $^{\mathrm{b}}$ and TEIICHIRO OGAWA ${ }^{\mathrm{b}, * *}$ \\ a SEDC Laboratory of Analytical Sciences, Department of Chemistry, \\ Xiamen University, Xiamen 361005, P.R. China; \\ ${ }^{\mathrm{b}}$ Department of Molecular Science and Technology, Kyushu University, \\ Kasuga-shi, Fukuoka 816, Japan
}

(Received 19 July 1997)

\begin{abstract}
A sensitive comparative fluorescence microscopic approach was developed and used to study the water at the air-water interface region. An insoluble chromophore meso$\alpha, \beta, \gamma, \delta$-tetraphenylporphine (TPP) was spread on the water. Its fluorescence spectrum indicated that the spectrum of TPP on the water surface was similar to those measured in nonpolar hydrocarbon solvents. Thus, the water at the surface was concluded to be hydrophobic and less polar than the water in the bulk.
\end{abstract}

Keywords: Polarity; hydrophobic; water surface; fluorescence; microscopy

\section{INTRODUCTION}

Liquid surfaces and interfaces are of fundamental scientific, technological, environmental and biological importance. Air-water interface is a key target for surface chemistry. Water at the surfaces and interfaces has different properties with the bulk water, and this difference has attracted many investigations $[1-10]$. It has, however,

\footnotetext{
${ }^{*}$ Present address: Department of Molecular Science and Technology, Kyushu University, Kasuga-shi, Fukuoka 816, Japan.

${ }^{* *}$ Corresponding author.
} 
been difficult to investigate the properties of air-water interfaces by the conventional spectroscopy. The second harmonic generation and the two-photon ionization have been successful to clarify several distinguished properties of the water surface [3-16], because these non-linear techniques have the special characteristic of being interface selective.

Fluorescence spectroscopy is sensitive and informative, but not interface-selective, because it has no intrinsic spatial resolution. The properties of monolayer or multilayer at the air-water interface have been investigated by fluorescence approaches [17], but this technique is not sensitive enough to measure small number of molecules below a monolayer coverage. Total internal reflection fluorescence can be used to investigate the liquid-solid and liquid-liquid interfaces at a high sensitivity, but not the air-liquid interfaces [18-19]. Spatial resolution can be improved by using a microscope. The best vertical resolution of the confocal microscopy [20-24] is, to our knowledge, ca. $05 \mu \mathrm{m}$, which would be larger than a thickness of the interfacial layer of several tens nanometer, and the confocal fluorescence microscopy does not meet the requirement for the study of interface by itself. A nearfield microscope has a higher spatial resolution; its probe, however, is so near to the analyte that the environment may be disturbed [25-29].

Because an insoluble molecule stays on the surface of water, an insoluble fluorescent molecule can be used as a probe to investigate the property of the air-water interface, even though the technique in itself has not enough spatial resolution of a monolayer thickness. In order to study properties of surface environment itself, the probe concentration should be lower than a monolayer coverage. In such a situation, a confocal microscopy is necessary and useful to remove effects of fluorescent impurities and residual probes in the bulk of the solution, which is overwhelmingly larger in quantity than the surface, for the investigation of a few probe molecules on the surface. The laser excitation and CCD detection offers enough sensitivity for a study of submonolayer coverage.

In the present study, we use a fluorescence microscope with a relatively low spatial resolution and an insoluble fluorescent probe to investigate environmental properties of the surface of water. An objective lens with a long working distance has allowed comparative experiments among different solvent environments. The polarity of 
water at the surface was found to be much smaller than expected. This comparative fluorescence microscopic approach may be extended to investigate other phenomena of the air-liquid interface.

\section{EXPERIMENTAL}

The experimental setup (Fig. 1) consists of a microscope (Nikon, Labophot-2), a CW argon ion laser (LEXEL, Model 95-4: 1.7 W for $514.5 \mathrm{~nm}$ ) and a CCD camera (SBIG, Model ST-6) with a
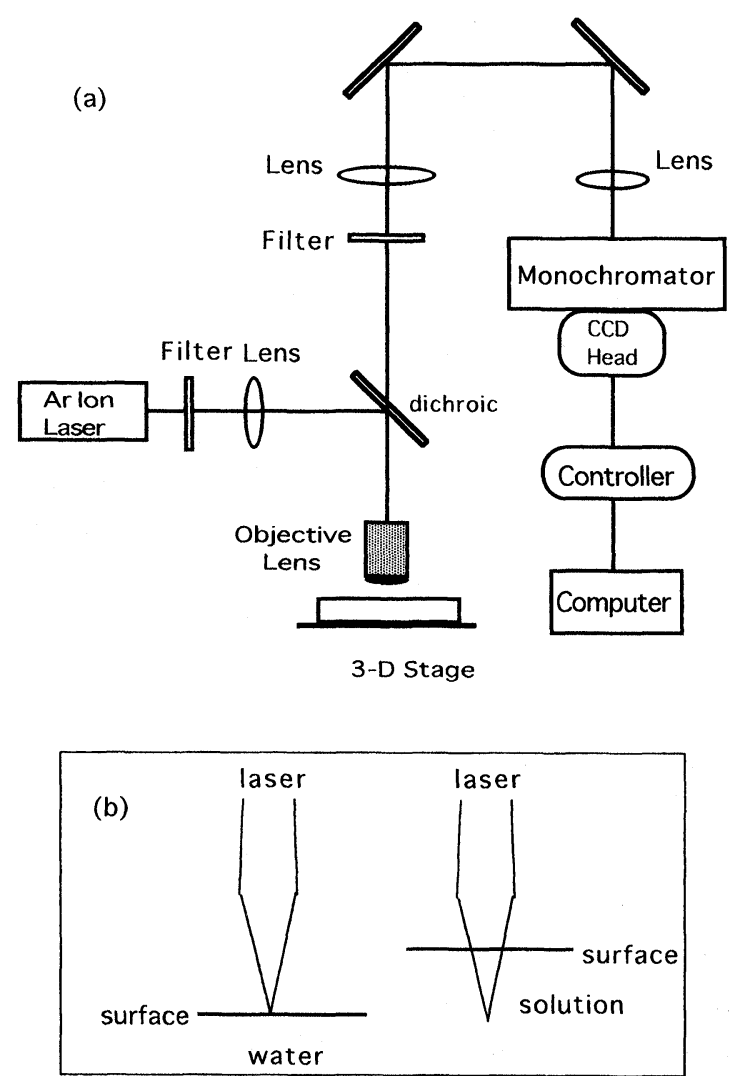

FIGURE 1 The schematic diagram of the experimental microscope setup (a) and the laser illumination to the surface (b). 
monochromator (Thermo Jurrell Ash, MonoSpec 18). A quartz sample cell with a diameter of $5 \mathrm{~cm}$ was placed on the stage of the microscope. The stage could be easily moved vertically or laterally by a manual drive or by a stepping motor to the accuracy of $1 \mu \mathrm{m}$. The working distance of the objective lens was long $(5.6 \mathrm{~mm})$ and the numerical aperture was low $(0.25)$. The laser beam $(514.5 \mathrm{~nm})$ was directed into the microscope objective by a dichroic beam splitter and focused to a spot on the surface or in the bulk of the solution in the cell. Fluorescence was collected by the same objective and was directed to the slit of the monochromator through the same beam splitter, an optial filter, lenses and mirrors. The slit $(100 \mu \mathrm{m}$ width) worked as a pinhole in the present confocal system. Fluorescence spectra were then recorded by the CCD camera. The fluorescence spectra at water surface and in various polar and nonpolar solvents were obtained by moving the microscope stage upwards and downwards. The fluorescence excitation and emission spectra were also obtained by a Hitachi F4010 spectrofluorimeter.

Meso- $\alpha, \beta, \gamma, \delta$-tetraphenylporphine (TPP) was purchased from Wako Pure Chemical Industries. Hexane was distilled and dehydrated with $\mathrm{CaCl}_{2}$. Water is deionized and double-distilled. All chemicals are special reagent grade.

TPP is insoluble in water [30]. A very small quantity of TPP was dissolved in organic solvents to prevent TPP to form aggregates and then was spread onto the water surface. The organic solvents evaporated rapidly and a loosely-arranged layer of TPP remained on the water surface.

\section{RESULTS AND DISCUSSION}

Fluorescence spectra of TPP in ethanol were measured by the conventional F4010 spectrofluorimeter, as shown in Figure 2; the spectra exhibited several excitation peaks and one emission peak. The laser beam at $514.5 \mathrm{~nm}$ was selected to excite the sub-band at 518 $\mathrm{nm}$, and the excitation at this wavelength provided fluorescence which is intense enough for observation. No fluorescence signal of TPP in water was observed from the bulk of the solution using the present 


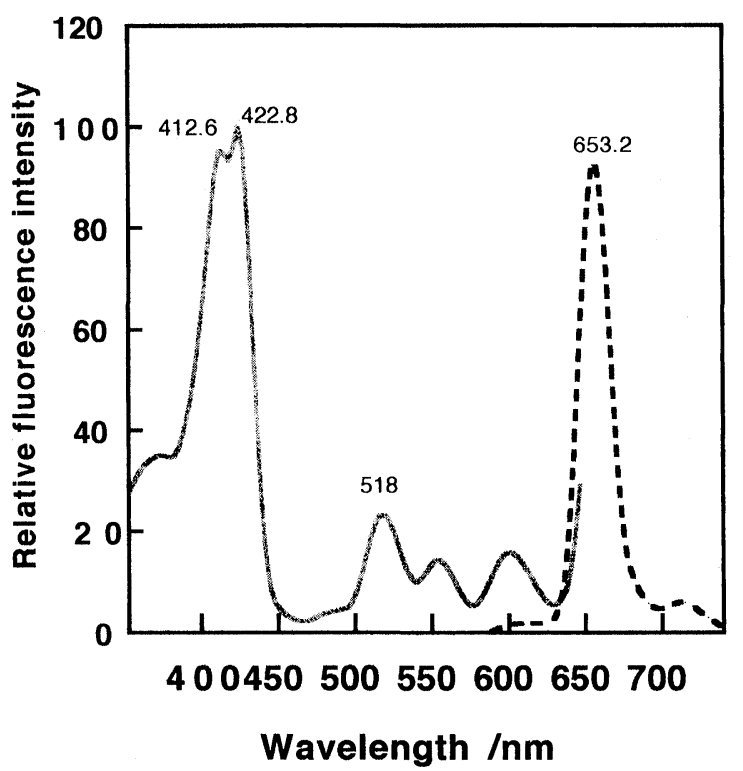

FIGURE 2 Fluorescence excitation (solid line) and emission spectra (broken line) of TPP (2 ppm in ethanol) obtained using conventional spectrofluorimeter.

confocal system. Thus, all the fluorescence of TPP measured in the present study should arise from TPP at the air-water interface [30].

The depth resolution of the present system was about $20 \mu \mathrm{m}$ at FWHM as indicated by the fluorescence intensity of TPP on water surface at various stage heights as shown in Figure 3; the abscissa is relative values, and the signal at $-40 \mu \mathrm{m}$ represents background. This depth resolution was not due to the depth distribution of TPP molecules but due to the relatively low spatial resolution of the system, because TPP was concentrated at the water surface. The fluorescence signal arose only from the surface of water. The high background indicated necessity of spatial resolution even by using an insoluble probe in order to remove any effects of residual probes and impurities in the bulk. In this situation the vertical adjustment of the stage was not serious.

The laser can be focused at any desired position to obtain the fluorescence emission spectra in the bulk or on the surface of the solution by moving the vertical position of stage as shown in Figure 


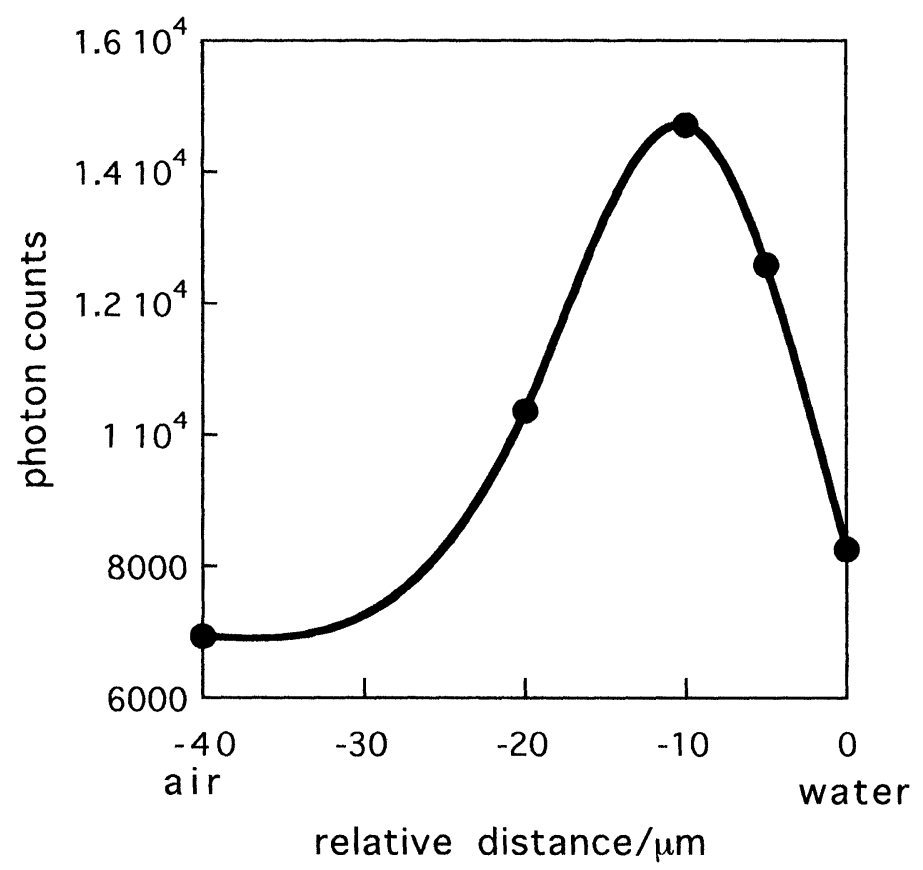

FIGURE 3 Depth profile of TPP $\left(8 \times 10^{-8} \mathrm{~g}\right)$ at water surface. The position 0 is arbitrary. The background was about 6000 counts.

1(b). Thus we can compare the fluorescence spectra of TPP in the bulk and on the surface using the same fluorescence microscope system.

Fluorescence spectra of TPP dissolved in various solvents were recorded. Each solvent offers a distinctive environment to solutes and plays a fundamental role in excitation and emission processes. All fluorescence spectra of TPP obtained in different solvents could distinctly be divided into two groups (Fig. 4(a)). The one group consists of TPP in polar solvents (ethanol, methanol, acetone) with a fluorescence peak at $646.6-647.3 \mathrm{~nm}$ and another weaker peak at $712.0-713.0 \mathrm{~nm}$. No apparent peak changes were observed among ethanol-water mixed solvents, and the spectral band shapes of TPP in all these polar solvents are almost identical. The other group consists of TPP in nonpolar solvents (benzene, hexane, cyclohexane, 2,2,4trimethylpentane) with a fluorescence peak at $649.7-651.0 \mathrm{~nm}$ and another weaker peak at $715.5-716.0 \mathrm{~nm}$. 

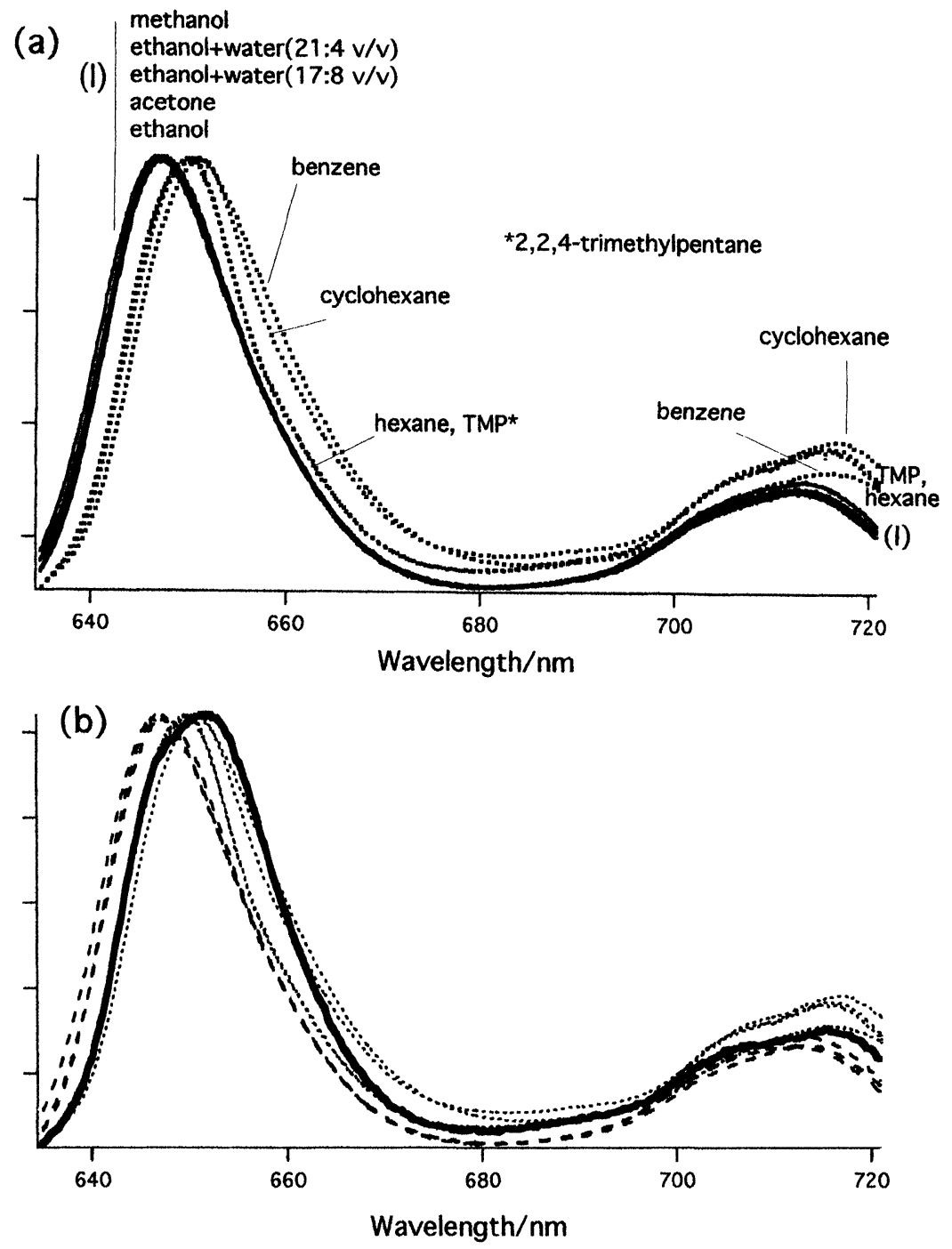

FIGURE 4 Normalized fluorescence spectra of TPP at various environments. (a) Spectra of TPP (2 ppm) in various polar solvents (methanol, ethanol, ethanol + water, acetone) are shown as solid lines and those in various nonpolar solvents (hexane, 2,2,4trimethypentane, cyclohexane, benzene) are shown as dotted lines. Laser power: 0.1 W. Integration time: $1 \mathrm{sec}$. (b) Spectrum of TPP $\left(4 \times 10^{-8} \mathrm{~g}\right.$ TPP $)$ at water surface is shown in heavy solid line. Laser power: $0.1 \mathrm{~W}$. Integration time: $30 \mathrm{sec}$. Those in polar and nonpolar solvents are shown in dashed and dotted lines as references; they are the same as in (a). 
The fluorescence spectra of TPP on water surface were interestingly found to belong to the second group as shown in Figure 4(b). The surface density of TPP was kept low enough to avoid mutual interactions and aggregations. As long as the amount of TPP onto water surface is small $\left(1-8 \times 10^{-8} \mathrm{~g}\right)$, the spectrum showed no concentration dependence. If we assume all TPP molecules stay in a single layer, the molecular area would be ca. $50 \mathrm{~nm}^{2} /$ molecule for $4 \times 10^{-8} \mathrm{~g}$ TPP since we have $3.9 \times 10^{13}$ molecules in total and the water surface area is $19.6 \mathrm{~cm}^{2}$. When the amount of TPP is higher than $10^{-6} \mathrm{~g}$, the spectrum changed dramatically and the peak at $651.5 \mathrm{~nm}$ was split into two peaks at $640 \mathrm{~nm}$ and $649 \mathrm{~nm}$, which should be due to the aggregation of TPP molecules. The surface fluorescence spectra did also not depend on the organic solvents which were used to solve TPP before spreading onto the water surface. Comparing Figures 4(a) and (b), the main and minor peaks of TPP are common at the air-water surface and in nonpolar solvents. Furthermore there is a weak but perceptible shoulder at ca. $705 \mathrm{~nm}$ for TPP in 2,2,4-trimethylpentane, hexane, cyclohexane though this band of TPP in benzene was not clear, while nothing can be found out for TPP in all polar solvents. Meaningfully, there is also such a shoulder on water surface. The fluorescence spectra of TPP at water surface are so similar to those in nonpolar solvent and much different from those in polar solvents.

A spectral shift of a molecule in different solvents reflects change in solvent property and environment. Therefore, the environment of water at surface should be similar to that of nonpolar solvents, i.e., the water is less polar and more hydrophobic on its surface than its bulk. the solvent polarity is the overall solvation capability of a solvent. The low density of water at surface, the asymmetry inherent to an interface, and broken hydrogen-bond network could weaken interaction between solute and water and could contribute to small polarity and hydrophobic character $[3,14]$. The surface is asymmetric in itself, and a molecule on surface faces to air, which has a small dielectric constant and should contribute hydrophobic character at surface. Several researches have indicated that the air-water interface is hydrophobic by using the interface-specific techniques such as surface two-photon ionization [10], second harmonic generation [3, 4, 8] and sum frequency generation [9]. The data based on different techniques have provided proofs of the nonpolar and hydrophobic 
character of water at surface. The present result has added another proof for it.

The fluorescence peak of TPP on the water surface is apparently wider than those in organic solvents as shown in Figure 4(b). This finding may indicate that the peak consists of a mixture of a few peaks, which may be due to a few types of TPP solvation on the water surface.

The property of the solvent at liquid interfaces is important in understanding chemical and physical processes at the interface. The comparative fluorescence confocal microscope method suggested in this paper could be used to extend such understandings by overcoming the limit of spatial resolution of fluorescence spectroscopy, and to investigate various aspects of liquid-air interfaces.

\section{Acknowledgements}

A JSPS postdoctoral fellowship of Japan Society for the Promotion of Science and a Grant-in-Aid (\#0532) from the Ministry of Education, Science and Culture to YQL are gratefully acknowledged.

\section{References}

[1] Fillingim, T. G., Zhu, S.-B., Yao, S. and Lee, J. (1989). Chem. Phys. Lett., 161, 444.

[2] Zhu, S.-B., Fillingim, T. G. and Robinson, G. W. (1991). J. Phys. Chem., 95, 1002.

[3] Bhattacharyya, K., Sitzmann, E. V. and Eisenthal, K. B. (1987). J. Chem. Phys., 87, 1442.

[4] Zhao, X. L., Subrahmanyan, S. and Eisenthal, K. B. (1990). J. Chem. Phys., 171, 558.

[5] Eisental, K. B. (1996). J. Phys. Chem., 100, 12997.

[6] Castro, A., Bhattacharyya, K. and Eisenthal, K. B. (1991). J. Chem. Phys., 95, 1310.

[7] Shi, X., Borguet, E., Tarnovsky, A. N. and Eisenthal, K. B. (1996). Chem. Phys., $205,167$.

[8] Wang, H., Borguet, E. and Eisenthal, K. B. (1997). J. Phys. Chem. A, 101, 713.

[9] Du, Q., Freysz, E. and Shen, Y. R. (1994). Science, 264, 826.

[10] Ogawa, T., Chen, H.-T., Inoue, T. and Nakashima, K. (1994). Chem. Phys. Lett., 229, 328.

[11] Sitzmann, E. V. and Eisenthal, K. B. (1988). J. Phys. Chem., 92, 4579.

[12] Zhuang, X., Lackritz, H. S. and Shen, Y. R. (1995). Chem. Phys. Lett., 246, 279.

[13] Sitzmann, E. and Eisenthal, K. B. (1989). J. Chem. Phys., 90, 2831.

[14] Castro, A., Sitzmann, E. V., Zhang, D. and Eisenthal, K. B. (1991). J. Phys. Chem., 95, 6752 .

[15] Meech, S. R. and Yoshihara, K. (1990). Chem. Phys. Lett., 174, 423.

[16] Chen, H.-T., Inoue, T. and Ogawa, T. (1994). Anal. Chem., 66, 4150. 
[17] Schmitz, P., Gruler, H. and Eberhardt, M. (1995). Mol. Cryst. Liq. Cryst., 262, 129.

[18] Yanagimachi, M., Tamai, N. and Masuhara, H. (1993). Chem. Phys. Lett., 201, 115.

[19] Watarai, H. and Saitoh, Y. (1995). Chem. Lett., 283.

[20] Dai, Y.-Q., Whittal, R. M. and Li, L. (1996). Anal. Chem., 68, 2494.

[21] Nie, S., Chiu, D. T. and Zare, R. N. (1995). Anal. Chem., 67, 2849.

[22] Kim, H.-B., Hayashi, M., Nakatani, K., Kitamura, N., Sasaki, K., Hotta, J.-I. and Masuhara, H. (1996). Anal. Chem., 68, 409.

[23] Delaney, P. M., Harris, M. R. and King, R. G. (1994). Applied Optics, 33, 573.

[24] Jacobsen, H., Hanninen, P., Soini, E. and Hell, S. W. (1994). J. Microsc., 176, 226.

[25] Trautman, J. K., Macklin, J. J., Brus, L. E. and Betzig, E. (1994). Nature, 369, 40.

[26] Higgins, D. A., Reid, P. J. and Barbara, P. F. (1996). J. Phys. Chem., 100, 1174.

[27] Betzig, E. and Chichester, R. J. (1993). Science, 262, 1422.

[28] Xie, X. S. and Dunn, R. C. (1994). Science, 265, 361.

[29] Macklin, J. J., Trautman, J. K., Harris, T. D. and Brus, L. E. (1996). Science, 272, 255.

[30] Martin, M. T., Prieto, I., Munoz, E., Camacho, L. and Avila, J. L. (1995). J. Colloid Interface Sci., 175, 83. 